\title{
A successful vaginal myomectomy of cervical leiomyoma in early pregnancy
}

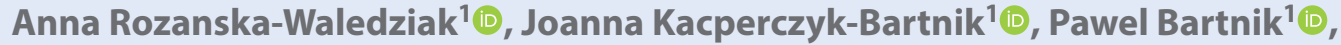 \\ Maciej Waledziak² ${ }^{\circledR}$, Krzysztof Czajkowski ${ }^{1}$ \\ ${ }^{1} 2^{\text {nd }}$ Department of Obstetrics and Gynecology, Medical University of Warsaw, Poland \\ 2Department of General, Oncological, Metabolic and Thoracic Surgery, Military Institute of Medicine, Warsaw, Poland
}

\begin{abstract}
The prevalence of clinically symptomatic cervical leiomyomas in pregnancy is lower than $0.1 \%$. Surgical intervention is necessary in extremely rare cases and only few are described in the literature. This study presents a case of successful vaginal myomectomy in the $13^{\text {th }}$ week of pregnancy followed by a delivery of a healthy neonate in term by cesarean section. Key words: gynecologic surgical procedure; leiomyoma; pregnancy trimester; first
\end{abstract}

Ginekologia Polska 2021; 92, 4:333-334

\section{INTRODUCTION}

About five percent of uterine leiomyomas are located in the cervix. Cervical leiomyomas rarely grow to an extent which causes symptoms, including lower abdominal pain, abnormal urination, dyspareunia and obstructed labor. Large leiomyomas can extend out through the external cervical os, leading to contact bleeding. The prevalence of clinically symptomatic cervical leiomyomas in pregnancy is lower than $0.1 \%$ with a necessity of precipitate intervention in extremely rare cases [1].

\section{CASE REPORT}

A 31-year-old woman with a history of three vaginal deliveries and no miscarriages was admitted to the clinic in the eighth gestational week because of lower abdominal pain. Vaginal examination showed cervical bleeding of low intensity and a smooth, firm, movable tumor located in the cervix. A vaginal ultrasound showed features of a benign leiomyoma, with dimensions of $76 \times 68 \times 62 \mathrm{~mm}$ and a live fetus of CRL $39.5 \mathrm{~mm}$ in the uterus with no sign of subchorionic hematoma. There were no symptoms of anemia in the blood count performed on the admission, with HGB $13.0 \mathrm{~g} / \mathrm{dL}, \mathrm{HCT} 39 \%, \mathrm{RBC}$ $4.57 \times 10^{6} / \mathrm{uL}$ and PLT $236 \times 10^{3} / \mathrm{uL}$. After receiving conservative treatment with analgetic drugs administered intravenously and then orally, the patient was discharged with no symptoms.

She was readmitted in the $13^{\text {th }}$ gestational week with severe abdominal pain and vaginal bleeding and was qualified for vaginal myomectomy in spinal anesthesia. The posterior wall of the cervix was incised and a total resection of a tumor of $10 \mathrm{~cm}$ in diameter was performed, with estimated blood loss of $200 \mathrm{~mL}$. The ultrasound examination after the surgery confirmed a live fetus. The pathological examination of the specimen confirmed a benign leiomyoma. There were no complications in the postoperative period and the patient was discharged in good general condition.

The patient was admitted to the clinic in the $40^{\text {th }}$ week of pregnancy with suspicious cardiotocography (CTG) tracings, including fetal tachycardia of 170 beats/min and increased variability. She was qualified for labor induction with oxytocin infusion. The patient reached $5 \mathrm{~cm}$ of dilation but was finally qualified for a cesarean section due to prolonged labor. A male neonate was born with birth weight of $3420 \mathrm{~g}$ and length of $53 \mathrm{~cm}, 9$ points Apgar. There were no complications during the postpartum period and the patient and her infant were discharged four days after the cesarean section. 


\section{DISCUSSION}

There are few reports in the literature considering cervical leiomyomas and prolapsed submucosal leiomyomas complicating pregnancies and therefore there are no management recommendations established. Early surgical intervention should be considered in case of hemorrhage, infection, pain or urinary stasis [1]. Vaginal myomectomy in pregnancy carries a risk of hemorrhage, spontaneous abortion, premature rupture of membranes/delivery and hysterectomy. Regarding the presented case, the surgery performed on the cervix may also lead to difficulties in cervix dilation during vaginal labor. Obara et al. presented a case of a partial resection of a cervical leiomyoma in the $13^{\text {th }}$ of pregnancy. Due to the thick base of the leiomyoma, they decided only to excise the myoma nucleus, having left a residual fragment of 3-4 cm of thickness. The patient had a vaginal delivery after oxytocin induction and mechanical dilatation of cervix in the $40^{\text {th }}$ week of pregnancy [2]. Kilpatrick et al. [3] described two cases of vaginal myomectomies in pregnancy. The first one was performed in the $15^{\text {th }}$ gestational week followed by a vaginal delivery in the $38^{\text {th }}$ week. The second patient underwent the procedure in the $13^{\text {th }}$ week of gestation, which was complicated by preterm premature rupture of membranes in the $16^{\text {th }}$ gestational week [3].

To conclude, symptomatic cervical leiomyomas are rare findings in pregnancy and require meticulous attention and intervention only in case of absolute necessity.

\section{Conflicts of interest}

The authors declare no conflict of interest.

\section{REFERENCES}

1. Straub HL, Chohan L, Kilpatrick CC. Cervical and prolapsed submucosal leiomyomas complicating pregnancy. Obstet Gynecol Surv. 2010; 65(9): 583-590, doi: 10.1097/OGX.0b013e3181fc5602, indexed in Pubmed: 21144089.

2. Obara M, Hatakeyama Y, Shimizu Y. Vaginal Myomectomy for Semipedunculated Cervical Myoma during Pregnancy. AJP Rep. 2014; 4(1): 37-40, doi: 10.1055/s-0034-1370352, indexed in Pubmed: 25032058.

3. Kilpatrick CC, Adler MT, Chohan L. Vaginal myomectomy in pregnancy: a report of two cases. South Med J. 2010; 103(10): 1058-1060, doi: 10.1097/SMJ.0b013e3181 efb552, indexed in Pubmed: 20818303. 